Revisão Sistemática

Systematic Review

Joice de Moura Silva ${ }^{1}$ (1)

Patricia Dominguez Campos ${ }^{1}$ (1)

Adriane Lima Mortari Moret ${ }^{1}$ (1)

Descritores

Qualidade de Vida

Criança

Perda Auditiva

Implante Coclear

Revisão

Keywords

Quality of Life

Child

Hearing Loss

Cochlear Implantation

Review

Endereço para correspondência:

Joice de Moura Silva

Rua Silvio Machione, 3-20, Bauru

(SP), Brasil, CEP: 17012-900

E-mail: joicemoura@live.com

Recebido em: Junho 13, 2019

Aceito em: Março 03, 2020

\section{Variáveis influenciadoras na qualidade de vida de crianças com implante coclear: revisão sistemática}

\author{
Influencing variables in the quality of life of \\ children with cochlear implants: a systematic \\ review
}

\section{RESUMO}

Objetivo: Verificar como a qualidade de vida de crianças com implante coclear foi avaliada em estudos publicados e analisar criticamente quais foram as variáveis influenciadoras. Estratégia de pesquisa: Pesquisa norteada pelas recomendações do PRISMA. A pergunta norteadora foi elaborada com base na estratégia PICO, sendo: "Quais variáveis são consideradas como influenciadoras sobre a qualidade de vida de crianças com implante coclear?". Os descritores selecionados foram: implante coclear, implantes cocleares, qualidade de vida, criança e seus sinônimos, nos idiomas português, inglês e espanhol. Foram identificados estudos indexados nas bases de dados: Pubmed/MEDLINE, LILACS, Scopus, SciELO, Embase, EBSCO/CINAHL e Web of Science. Critérios de seleção: Foram selecionados estudos direcionados ao tema, com níveis de evidência científica de 1 a 4 , publicados nos idiomas português, inglês e espanhol, no período de 2009 a 2018. Análise dos dados: inicialmente analisou-se os títulos de todos os estudos encontrados, seguido dos resumos e da leitura na íntegra dos textos mais relevantes. Resultados: Após a análise rigorosa dos 1062 artigos, oito foram classificados como contendo as respostas necessárias à pergunta norteadora de pesquisa. Os estudos selecionados foram publicados entre os anos de 2009 a 2016, classificados em nível de evidência 2a e 4, com casuística entre dez a 259 crianças e com idades entre 18 meses a 18 anos incompletos. Conclusão: Os fatores influenciadores que se correlacionaram com a qualidade de vida das crianças com implante coclear foram: a implantação precoce, o uso do dispositivo eletrônico, as habilidades auditivas e as habilidades de linguagem.

\begin{abstract}
Purpose: To verify how the quality of life of children with cochlear implant was evaluated in published studies and to critically analyze which were the influencing variables. Research Strategy: Research guided by PRISMA recommendations. The guiding question was elaborated based on the PICO strategy, being: "Which variables are considered as influencers on the quality of life of children with cochlear implants?" The selected descriptors were cochlear implants, cochlear implants, quality of life, child and their synonyms, in the Portuguese, English and Spanish languages. We have identified indexed studies in the databases: Pubmed / MEDLINE, LILACS, Scopus, SciELO, Embase, EBSCO / CINAHL and Web of Science. Selection Criteria: Selected studies were selected, with levels of scientific evidence from 1 to 4, published in the Portuguese, English and Spanish languages, from 2009 to 2018. Data analysis: initially the titles of all the studies, followed by summaries and full reading of the most relevant texts. Results: After the rigorous analysis of the 1062 articles, eight were classified as containing the necessary answers to the guiding question of research. The selected studies were published between 2009 and 2016, classified as evidence level 2a and 4, with a sample of between 10 and 259 children and between the ages of 18 months and 18 years. Conclusion: The influencing factors that correlated with the quality of life of children with cochlear implants were early implantation, use of the electronic device, hearing skills and language skills.
\end{abstract}

Trabalho realizado na Faculdade de Odontologia de Bauru - FOB, Universidade de São Paulo - USP - Bauru (SP), Brasil.

${ }^{1}$ Faculdade de Odontologia de Bauru, Universidade de São Paulo - USP - Bauru (SP), Brasil.

Fontes de financiamento: Coordenação de Aperfeiçoamento de Pessoal de Nível Superior - Brasil (CAPES) Código de Financiamento 001 e Fundação de Amparo à Pesquisa do Estado de São Paulo (FAPESP) - Número de Processo: 2016/24216-0.

Conflito de interesses: nada a declarar. 


\section{INTRODUÇÃO}

As perdas auditivas bilaterais de grau severo e/ou profundo ocorridas na infância, em especial na fase pré-lingual, restringem de forma potencial o desenvolvimento comunicativo com prejuízos significativos sobre as áreas psicossocial, cognitiva, emocional, acadêmica, além da qualidade de vida desta população ${ }^{(1,2)}$.

O implante coclear (IC) é considerado um importante recurso tecnológico altamente efetivo na habilitação de crianças com deficiência auditiva pré-lingual que não apresentam benefícios com o uso do aparelho de amplificação sonora individual (AASI) ${ }^{(2-4)}$. Entretanto, a cirurgia e a adaptação do IC isoladamente não garantem o pleno benefício de seus usuários. Diversas variáveis podem interferir no desempenho e na qualidade de vida das crianças implantadas, tais como: a etiologia, a idade na cirurgia e na ativação do IC, o tempo de privação sensorial auditiva, o resíduo auditivo pré-operatório, o número de eletrodos inseridos na cóclea, o tempo de uso diário do dispositivo, a inserção em terapia fonoaudiológica especializada baseada na abordagem aurioral e o envolvimento familiar no processo terapêutico ${ }^{(4-10)}$.

Essa gama de variabilidades torna o processo de implantação complexo, multidimensional e gera desafios na compreensão dos motivos pelos quais algumas crianças alcançam melhores resultados em testes de avaliação, de desenvolvimento, e de qualidade de vida, quando comparadas à evolução de outras crianças com os mesmos critérios de indicação e adaptação do $\mathrm{IC}^{(4,6,11,12)}$.

Para garantir melhores resultados de desenvolvimento em crianças com deficiência auditiva estudos sugerem que o processo terapêutico com dispositivos eletrônicos seja iniciado de forma precoce, associado à medidas adequadas de habilitação com terapia fonoaudiológica especializada, incrementadas com planejamentos intensos de orientação e aconselhamento familiar. Esta premissa acompanhada de outras variáveis pode influenciar o desenvolvimento das habilidades auditivas, o processo de aquisição e desenvolvimento da comunicação falada, além da inserção e participação em diversos ambientes, com melhores resultados sobre a autoconfiança, a autonomia, o bem-estar, a felicidade, o processos de escolarização, a socialização e a qualidade de vida destas crianças e suas famílias ${ }^{(4,6,10,11,13-15)}$.

\section{OBJETIVO}

O presente estudo teve como objetivo realizar uma revisão sistemática pautada em verificar como a qualidade de vida de crianças com implante coclear foi avaliada em estudos publicados e analisar criticamente quais foram as variáveis influenciadoras.

\section{ESTRATÉGIA DE PESQUISA}

A presente revisão sistemática foi conduzida de acordo com os critérios orientados pela biblioteca Cochrane Handbook ${ }^{(16)}$, baseado no método PRISMA (Prefered Reporting Items for Systematic Reviews and Meta-Analyses) ${ }^{(17,18)}$, o qual recomenda a utilização das seguintes etapas: (I) identificação, (II) seleção, (III) elegibilidade e (IV) inclusão.

A questão norteadora responsável por conduzir as estratégias de buscas direcionadas aos estudos com elevada evidência científica, foi elaborada com base no tema de pesquisa e orientada pela estratégia PICO (Population, Intervention, Comparation, Outcome). Assim, estabeleceu-se a seguinte pergunta: Quais variáveis são consideradas como influenciadoras sobre a qualidade de vida de crianças com implante coclear?

Para a busca dos artigos foram selecionados os descritores e sinônimos listados no vocabulário de indexação da PubMed, Medical Subject Headings (MeSH terms) e na biblioteca de Descritores em Ciência da Saúde (DeCS), nos idiomas: português, inglês e espanhol (Quadro 1).

A Tabela 1 apresenta as estratégias de busca avançada utilizadas na pesquisa dos artigos, organizadas a partir das combinações dos descritores e sinônimos por meio do operador boleado "AND", e a quantidade de estudos identificados (etapa I) no levantamento bibliográfico em cada uma das bases de dados eletrônicas selecionadas e acessadas: Pubmed/MEDLINE, LILACS, Scopus, SciELO, Embase, EBSCO/CINAHL e Web of Science.

\section{CRITÉRIOS DE SELEÇÃO}

A seleção dos estudos (etapa II) foi conduzida por duas pesquisadoras de forma independente e cega, seguindo os

Quadro 1. Descritores e sinônimos elegidos para a busca bibliográfica.

\begin{tabular}{|c|c|c|c|c|c|}
\hline \multicolumn{2}{|c|}{ Português } & \multicolumn{2}{|c|}{ Inglês } & \multicolumn{2}{|c|}{ Espanhol } \\
\hline Descritores & Sinônimos & Descritores & Sinônimos & Descritores & Sinônimos \\
\hline \multirow[b]{2}{*}{ Implante Coclear } & Implantação coclear & \multirow[b]{2}{*}{ Cochlear implantation } & $\begin{array}{c}\text { Cochlear } \\
\text { implantations }\end{array}$ & \multirow[b]{2}{*}{ Implantación coclear } & \multirow[b]{2}{*}{$\begin{array}{l}\text { Implantación de } \\
\text { prótesis coclear }\end{array}$} \\
\hline & $\begin{array}{l}\text { Implante de prótese } \\
\text { coclear }\end{array}$ & & Cochlear prosthesis & & \\
\hline \multirow{3}{*}{ Implantes cocleares } & \multirow{3}{*}{-} & \multirow{3}{*}{ Cochlear implants } & Cochlear implant & \multirow{3}{*}{ Implantes cocleares } & \multirow{3}{*}{-} \\
\hline & & & & & \\
\hline & & & Cochlear prostheses & & \\
\hline Qualidade de vida & - & Quality of life & - & Calidad de vida & - \\
\hline \multirow{3}{*}{ Criança } & \multirow{3}{*}{ Crianças } & \multirow{3}{*}{ Child } & \multirow{3}{*}{ Children } & Niño & \multirow{3}{*}{ - } \\
\hline & & & & & \\
\hline & & & & Niños & \\
\hline
\end{tabular}


Quadro 2. Níveis de evidência científica de acordo com os critérios propostos pela ASHA.

\begin{tabular}{|c|c|}
\hline Níveis de evidência & Tipos de estudo \\
\hline $1 \mathrm{a}$ & Revisão sistemática ou meta-análise de alta qualidade de ensaios randomizados controlados \\
\hline $1 \mathrm{~b}$ & Ensaios controlados randomizados de alta qualidade \\
\hline $2 \mathrm{a}$ & Revisão sistemática ou meta-análise de alta qualidade de ensaios controlados não randomizados \\
\hline $2 \mathrm{~b}$ & Revisão sistemática de estudos de coorte \\
\hline $3 \mathrm{a}$ & Estudos de coorte individual ou ensaios controlados randomizados de baixa qualidade \\
\hline $3 \mathrm{~b}$ & Estudos de resultados clínicos \\
\hline 4 & Revisão sistemática de estudo de caso controle \\
\hline $5 \mathrm{a}$ & Estudo de caso controle individual \\
\hline $5 \mathrm{~b}$ & Série de casos \\
\hline 6 & Opinião de especialistas sem avaliação crítica explícita \\
\hline 7 &
\end{tabular}

Fonte: $\mathrm{ASHA}^{(19)}$.

Tabela 1. Total de artigos selecionados a partir das estratégias de busca e base de dados escolhidos para a pesquisa bibliográfica.

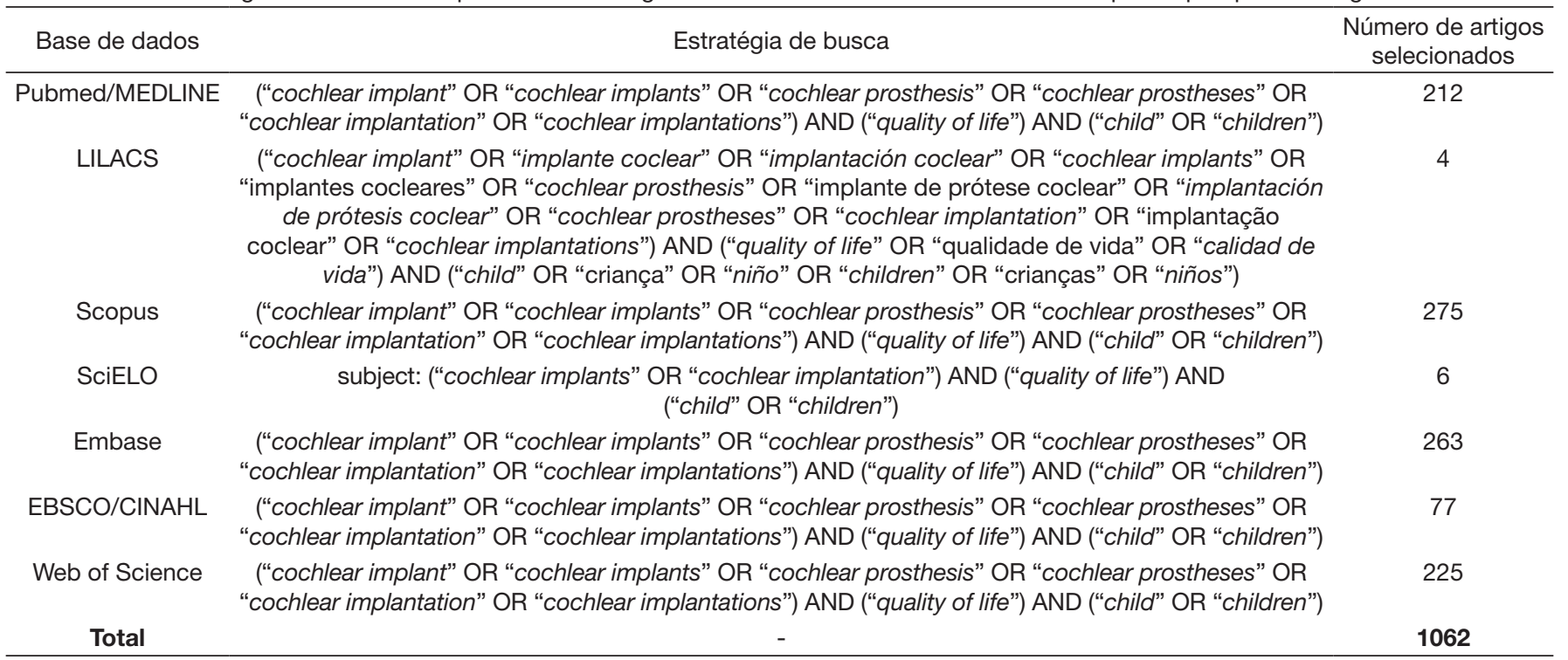

seguintes critérios inclusão: 1) população: crianças com implante coclear; 2) intervenção: implante coclear e habilitação ou reabilitação auditiva por método aurioral; 3) comparação: qualidade de vida após o uso do implante coclear; 4) resultados: variáveis de influência sobre a qualidade de vida de crianças com implante coclear; 5) tempo: publicações realizadas entre os anos de 2009 a 2018; 6) língua: português, inglês e espanhol; 7) tipos de estudos: pesquisas com níveis de evidência científica 1a, 1b, 2a, 2b, 3a, 3b, e 4, propostos pela American Speech and Hearing Association (ASHA) ${ }^{(19)}$ para o curso de fonoaudiologia (Quadro 2).

Foram excluídos: 1) estudos repetidos; 2) capítulos de livro; 3) estudos com resumo ou artigo na íntegra incompletos; 4) estudos que não abordavam a temática proposta; 5) estudos realizados com sujeitos maiores de 18 anos; 6) estudos realizados com crianças com Desordem do Espectro da Neuropatia Auditiva (DENA), hipoplasia do nervo auditivo, malformação da orelha externa, média ou interna, surdez unilateral, crianças não oralizadas ou com outros comprometimentos associados à deficiência auditiva; 7) estudos realizados com crianças não usuárias de IC; 8) estudos realizados com crianças adaptadas somente com IC bilateral simultâneo; 9) estudos com o objetivo principal de investigação da qualidade de vida e/ou comparação entre grupos (idosos, adultos, usuários de outros dispositivos eletrônicos, crianças não oralizadas, crianças com audição normal), sem o objetivo de investigar as variáveis influenciadoras sobre o desenvolvimento do grupo de crianças com IC; 10) estudos com resultados adquiridos unicamente a partir de impressões subjetivas sem dados objetivos e protocolos padronizados.

\section{ANÁLISE DOS DADOS}

Os dados levantados por meio das etapas de identificação (I) e seleção (II), foram analisados na etapa da elegibilidade (III). Inicialmente investigou-se os títulos de todos os estudos selecionados. Em seguida, os resumos dos artigos restantes da primeira etapa foram analisados quanto aos critérios de inclusão e exclusão previamente estabelecidos. Na etapa de inclusão (IV), após o estudo dos títulos e resumos, os textos que deixaram dúvidas quanto aos critérios de elegibilidade foram lidos na íntegra. A Figura 1 representa o detalhamento do processo de seleção das publicações incluídas na revisão sistemática da literatura. 


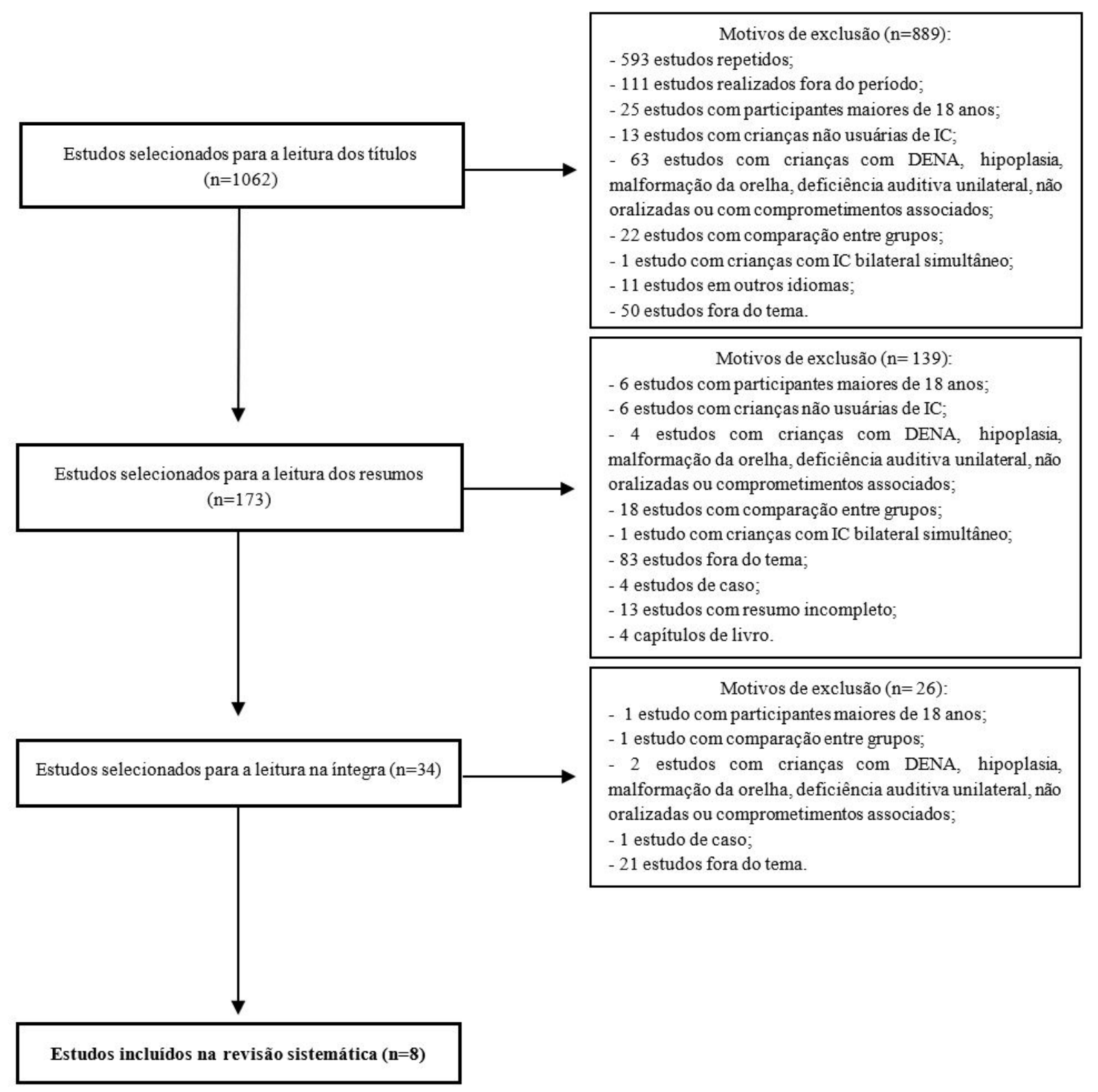

Figura 1. Seleção das publicações incluídas na revisão sistemática.

\section{RESULTADOS}

A partir da leitura rigorosa dos 34 estudos elegidos para a leitura na íntegra na etapa de inclusão (IV), oito artigos foram classificados como contendo as respostas necessárias à pergunta de pesquisa.

O detalhamento das características dos artigos incluídos na revisão sistemática da literatura em relação aos autores, título, nível de evidência cientifica, casuística e faixa etária, objetivos, protocolos utilizados e variáveis avaliadas, ocorreu por meio de uma ficha protocolar padronizada utilizada a fim de facilitar a análise dos dados e resgatar os detalhes relevantes à pesquisa (Tabela 2).

Os oito artigos selecionados foram publicados entre os anos de 2009 a 2016 e classificados em nível de evidência 4, com exceção da inclusão de uma revisão sistemática com nível de evidência 2a. Em pesquisas clínicas voltadas ao campo da audiologia poucos são os estudos que apresentam os desenhos preconizados para uma revisão sistemática (níveis 1 e 2). A casuística da revisão sistemática variou de dez a 259 crianças, de ambos os sexos, com idades entre 18 meses a 18 anos incompletos. No que se refere à qualidade metodológica, todos os trabalhos fizeram uso de ferramentas validadas para estudar a qualidade de vida de crianças com implante coclear e as variáveis influenciadoras.

De acordo com a Organização Mundial da Saúde (OMS) (20), a qualidade de vida diz respeito "a percepção do indivíduo de sua inserção na vida no contexto da cultura e sistemas de valores nos quais ele vive e em relação aos seus objetivos, expectativas, padrões e preocupações". Tal definição indica a complexa relação existente entre os aspectos multifatoriais que envolvem o bem estar físico, mental, psicológico, emocional 
Tabela 2. Ficha protocolar com as características dos estudos incluídos na revisão sistemática.

\begin{tabular}{|c|c|c|c|c|c|c|}
\hline Autores & Título & Nível de evidência & $\begin{array}{l}\text { Casuística e } \\
\text { faixa etária }\end{array}$ & Objetivos & $\begin{array}{l}\text { Protocolos } \\
\text { utilizados }\end{array}$ & Variáveis avaliadas \\
\hline $\begin{array}{l}\text { Liu, Liu, Kang, Gu, } \\
\text { Hong }\end{array}$ & $\begin{array}{l}\text { Evaluation on } \\
\text { health-related } \\
\text { quality of life in } \\
\text { deaf children with } \\
\text { cochlear implant } \\
\text { in China }\end{array}$ & Nível 4 & $\begin{array}{l}259 \text { crianças } \\
\text { com idades } \\
\text { entre } 48 \\
\text { meses a } 11 \\
\text { anos e seus } \\
\text { respectivos pais }\end{array}$ & $\begin{array}{c}\text { Avaliar as mudanças } \\
\text { na qualidade de } \\
\text { vida de crianças e } \\
\text { adolescentes com IC } \\
\text { unilateral usando o } \\
\text { HUI3 e NCIQ }\end{array}$ & $\begin{array}{c}\text { Questionários de } \\
\text { qualidade de vida: } \\
\text { HUI3, NCIQ; Teste } \\
\text { de percepção } \\
\text { auditiva da fala: } \\
\text { MSP'M }\end{array}$ & $\begin{array}{l}\text { Habilidades } \\
\text { auditivas }\end{array}$ \\
\hline $\begin{array}{c}\text { Noble, Hedley- } \\
\text { Williams, } \\
\text { Sunderhaus, } \\
\text { Dawant, Labadie, } \\
\text { Camarata, } \\
\text { Gifford(22) }^{(2)^{2}}\end{array}$ & $\begin{array}{l}\text { Initial results with } \\
\text { image-guided } \\
\text { cochlear implant } \\
\text { programming in } \\
\text { children }\end{array}$ & Nível 4 & $\begin{array}{l}18 \text { crianças com } \\
\text { idades entre } 5 \text { a } \\
17 \text { anos e seus } \\
\text { respectivos pais }\end{array}$ & $\begin{array}{c}\text { Verificar se a } \\
\text { programação do IC } \\
\text { guiada por imagem } \\
\text { pode melhorar } \\
\text { os resultados } \\
\text { da audição para } \\
\text { crianças com IC }\end{array}$ & $\begin{array}{l}\text { Questionário de } \\
\text { qualidade de vida: } \\
\text { PedsQL; Teste } \\
\text { de percepção } \\
\text { auditiva da fala: } \\
\text { LNT; Programação } \\
\text { do IC: IGCIP }\end{array}$ & $\begin{array}{l}\text { Habilidades } \\
\text { auditivas }\end{array}$ \\
\hline $\begin{array}{l}\text { Almeida, Matas, } \\
\text { Couto, Carvalho }\end{array}$ & $\begin{array}{c}\text { Quality of life } \\
\text { evaluation in } \\
\text { children with } \\
\text { cochlear implants }\end{array}$ & Nível 4 & $\begin{array}{l}15 \text { crianças com } \\
\text { idades entre } 2 \text { a } \\
12 \text { anos e seus } \\
\text { respectivos pais }\end{array}$ & $\begin{array}{l}\text { Avaliar a qualidade } \\
\text { de vida de } 15 \\
\text { crianças após } \\
\text { ativação do IC e } \\
\text { analisar as possíveis } \\
\text { correlações entre os } \\
\text { diferentes domínios } \\
\text { de qualidade de } \\
\text { vida, o tempo } \\
\text { de uso do IC e o } \\
\text { desenvolvimento } \\
\text { das habilidades } \\
\text { auditivas }\end{array}$ & $\begin{array}{l}\text { Questionário de } \\
\text { qualidade de vida: } \\
\text { CCIPP; Testes } \\
\text { de percepção } \\
\text { auditiva da fala: } \\
\text { GASP e Escala } \\
\text { de Categorias de } \\
\text { Audição }\end{array}$ & $\begin{array}{c}\text { Fator demográfico } \\
\text { (idade auditiva) } \\
\text { e habilidades } \\
\text { auditivas }\end{array}$ \\
\hline $\begin{array}{c}\text { Kumar, Warner- } \\
\text { Czyz, Silver, Betty, } \\
\text { Tobey }^{(24)}\end{array}$ & $\begin{array}{l}\text { American parent } \\
\text { perspectives on } \\
\text { quality of life in } \\
\text { pediatric cochlear } \\
\text { implant recipients }\end{array}$ & Nível 4 & $\begin{array}{l}32 \text { crianças com } \\
\text { idades entre } 4 \\
\text { a } 8 \text { anos e seus } \\
\text { respectivos pais }\end{array}$ & $\begin{array}{l}\text { Avaliar a qualidade } \\
\text { de vida de crianças } \\
\text { usuárias de IC } \\
\text { e correlacionar } \\
\text { com as variáveis } \\
\text { demográficas }\end{array}$ & $\begin{array}{c}\text { Questionário de } \\
\text { qualidade de vida: } \\
\text { CCIPP }\end{array}$ & $\begin{array}{c}\text { Fatores } \\
\text { demográficos } \\
\text { (idade } \\
\text { cronológica, idade } \\
\text { na ativação e } \\
\text { idade auditiva) }\end{array}$ \\
\hline $\begin{array}{c}\text { Morettin, Santos, } \\
\text { Stefanini, Antonio, } \\
\text { Bevilacqua, } \\
\text { Cardoso(25) }\end{array}$ & $\begin{array}{l}\text { Measures of } \\
\text { quality of life in } \\
\text { children with } \\
\text { cochlear implant: } \\
\text { systematic review }\end{array}$ & Nível 2a & $\begin{array}{c}\text { Estudos } \\
\text { realizados com } \\
\text { amostras de } 28 \\
\text { a } 222 \text { crianças } \\
\text { com idades } \\
\text { entre } 2 \text { a } 16 \\
\text { anos e seus } \\
\text { respectivos pais }\end{array}$ & $\begin{array}{l}\text { Identificar estudos } \\
\text { sobre a qualidade } \\
\text { de vida em crianças } \\
\text { usuárias de implante } \\
\text { coclear, levantar os } \\
\text { principais aspectos } \\
\text { avaliados nessa } \\
\text { população e os } \\
\text { fatores relacionados } \\
\text { com a medida da } \\
\text { qualidade de vida }\end{array}$ & $\begin{array}{l}\text { Questionários } \\
\text { de qualidade } \\
\text { de vida: CCIPP, } \\
\text { KINDL, EQ-5D, } \\
\text { CIF, questionário } \\
\text { específico para } \\
\text { avalição do IC } \\
\text { construído pelos } \\
\text { autores e VAS }\end{array}$ & $\begin{array}{c}\text { Fatores } \\
\text { demográficos } \\
\text { (idade na cirurgia, } \\
\text { idade cronológica, } \\
\text { e idade auditiva), } \\
\text { habilidades } \\
\text { auditivas e } \\
\text { habilidades de } \\
\text { linguagem }\end{array}$ \\
\hline $\begin{array}{c}\text { Fortunato-Tavares, } \\
\text { Befi-Lopes, Bento, } \\
\text { Andrade }^{(26)}\end{array}$ & $\begin{array}{c}\text { Children with } \\
\text { cochlear implants: } \\
\text { communication } \\
\text { skills and quality } \\
\text { of life }\end{array}$ & Nível 4 & $\begin{array}{l}10 \text { crianças com } \\
\text { idades entre } 4 \\
\text { a } 8 \text { anos e seus } \\
\text { respectivos pais }\end{array}$ & $\begin{array}{c}\text { Tradução e } \\
\text { adaptação de } \\
\text { um questionário } \\
\text { internacional para o } \\
\text { Português Brasileiro; } \\
\text { análise das } \\
\text { correlações entre } \\
\text { fatores relacionados } \\
\text { à qualidade de } \\
\text { vida; análise das } \\
\text { correlações entre } \\
\text { qualidade de vida e } \\
\text { medidas clínicas de } \\
\text { resultado }\end{array}$ & $\begin{array}{l}\text { Questionário } \\
\text { de qualidade } \\
\text { de vida: CCIPP; } \\
\text { Questionários de } \\
\text { linguagem: LAVE, } \\
\text { MUSS; Testes } \\
\text { de percepção } \\
\text { auditiva da fala: } \\
\text { MAIS, IT-MAIS }\end{array}$ & $\begin{array}{l}\text { Habilidades } \\
\text { auditivas e } \\
\text { habilidades de } \\
\text { linguagem }\end{array}$ \\
\hline
\end{tabular}

\footnotetext{
Legenda - IC= Implante coclear; AASI= Aparelho de Amplificação Sonora Individual; HUI3= Health Utilities Index Mark 3; NCIQ= Nijmegen Cochlear Implant Questionnaire; MSPTM= Mandarin Speech Perception test materials; PedsQL= Pediatric Quality of Life Inventory or; LNT= Lexical Neighborhood Test; IGCIP= Image-Guided Cochlear Implant Programming; CCIPP= Children with Cochlear Implants: Parental Perspectives; GASP = Glendonald Auditory Screening Perception; KINDL= Questionnaire Measuring Health-Related Quality of Life in Children and Adolescents; EQ-5D: Euro Quality of Life Instrument - 5D; CIF= Classificação Internacional de Funcionalidade, Incapacidade e Saúde; VAS= Visual Analogue Scale; LAVE= Lista de Avaliação Vocabulário Expressivo; MAIS= Meaningful Auditory Integration Scale; IT-MAIS= The Infant-Toddler Meaningful Auditory Integration Scale; MUSS= Meaningful Use of Speech Scale; SSQ= Speech, Spatial and Qualities of Hearing Scale for Parents; MLNT= Multisyllabic, Lexical Neighborhood Test; The QoL- C= The children's quality of life questionnaire.
} 
Tabela 2. Continuação...

\begin{tabular}{|c|c|c|c|c|c|c|}
\hline Autores & Título & Nível de evidência & $\begin{array}{l}\text { Casuística e } \\
\text { faixa etária }\end{array}$ & Objetivos & $\begin{array}{l}\text { Protocolos } \\
\text { utilizados }\end{array}$ & Variáveis avaliadas \\
\hline $\begin{array}{l}\text { Schorr, Roth, } \\
\text { Fox }^{(28)}\end{array}$ & $\begin{array}{l}\text { Quality of Life } \\
\text { for children with } \\
\text { cochlear implants: } \\
\text { perceived benefits } \\
\text { and problems and } \\
\text { the perception of } \\
\text { single words and } \\
\text { emotional sounds }\end{array}$ & Nível 4 & $\begin{array}{c}37 \text { crianças com } \\
\text { idades entre } 5 \text { a } \\
14 \text { anos }\end{array}$ & $\begin{array}{c}\text { Examinar as } \\
\text { percepções } \\
\text { subjetivas das } \\
\text { crianças sobre } \\
\text { a qualidade de } \\
\text { vida com o uso } \\
\text { do IC, investigar } \\
\text { a capacidade de } \\
\text { percepção da } \\
\text { emoção vocal e } \\
\text { a influência do } \\
\text { funcionamento } \\
\text { auditivo e da idade } \\
\text { na cirurgia do IC } \\
\text { sobre a qualidade } \\
\text { de vida }\end{array}$ & $\begin{array}{l}\text { Questionário de } \\
\text { qualidade de vida: } \\
\text { The QoL-C; Testes } \\
\text { de percepção } \\
\text { auditiva da fala: } \\
\text { LNT, MLNT, } \\
\text { Vocal emotion } \\
\text { identification }\end{array}$ & $\begin{array}{c}\text { Fatores } \\
\text { demográficos } \\
\text { (sexo, nível } \\
\text { socioeconômico, } \\
\text { idade cronológica, } \\
\text { idade na } \\
\text { adaptação do } \\
\text { AASI, idade na } \\
\text { ativação do IC e } \\
\text { idade auditiva), } \\
\text { habilidades } \\
\text { auditivas e } \\
\text { habilidades de } \\
\text { linguagem }\end{array}$ \\
\hline
\end{tabular}

Legenda - IC= Implante coclear; AASI= Aparelho de Amplificação Sonora Individual; HUI3= Health Utilities Index Mark 3; NCIQ= Nijmegen Cochlear Implant Questionnaire; MSPTM = Mandarin Speech Perception test materials; PedsQL= Pediatric Quality of Life Inventory or; LNT= Lexical Neighborhood Test; IGCIP= Image-Guided Cochlear Implant Programming; CCIPP= Children with Cochlear Implants: Parental Perspectives; GASP = Glendonald Auditory Screening Perception; KINDL= Questionnaire Measuring Health-Related Quality of Life in Children and Adolescents; EQ-5D: Euro Quality of Life Instrument - 5D; CIF= Classificação Internacional de Funcionalidade, Incapacidade e Saúde; VAS= Visual Analogue Scale; LAVE= Lista de Avaliação Vocabulário Expressivo; MAIS= Meaningful Auditory Integration Scale; IT-MAIS= The Infant-Toddler Meaningful Auditory Integration Scale; MUSS= Meaningful Use of Speech Scale; SSQ= Speech, Spatial and Qualities of Hearing Scale for Parents; MLNT = Multisyllabic, Lexical Neighborhood Test; The QoL- C= The children's quality of life questionnaire.

e espiritual, os relacionamentos sociais, familiares e afetivos, à saúde, à escola e a educação, à habitação, às experiências passadas, atuais e futuras dos indivíduos frente aos contextos culturais e valores éticos e morais, e à outras circunstancias da vida ${ }^{(15)}$.

Em crianças com implante coclear, além das dimensões acima discutidas, o estudo da qualidade de vida abrange também uma gama de aspectos relativos à autoestima, à autoimagem, e a autoconfiança construídas frente ao diagnóstico de deficiência auditiva, à satisfação com o uso do IC, à independência, à autossuficiência, o autocuidado, à mobilidade, à dor, o uso do celular e de dispositivos auxiliares da audição, às preferencias, à percepção e a compreensão auditivas da fala, à comunicação, e ainda, a atitude dos outros frente à todos estes fatores ${ }^{(25,26)}$. Na presente revisão sistemática, nota-se a investigação dos aspectos da qualidade de vida física, psicológica, emocional e social de crianças com implante coclear, influenciados preferencialmente pelas variáveis demográficas, as habilidades auditivas e as habilidades de linguagem.

As variáveis demográficas influenciadoras na qualidade de vida foram identificadas em quatro estudos ${ }^{(23-25,28)}$. $\mathrm{Na}$ análise entre o sexo, o nível socioeconômico, a idade cronológica, a idade na adaptação do AASI, a idade na ativação do IC, a idade auditiva (tempo de uso dos dispositivos eletrônicos), e a qualidade de vida realizada no primeiro estudo ${ }^{(27)}$, a variável idade no uso da primeira amplificação (AASI) revelou correlação negativa estatisticamente significante com a qualidade de vida, reforçando que, quanto menor a idade na adaptação do AASI, maior é a de qualidade de vida. Levando em conta que, as crianças que receberam o AASI mais precocemente possuíam, também, o maior tempo de uso do dispositivo, as maiores idades auditivas se correlacionaram de forma estatisticamente significante positiva com os mais altos índices de qualidade de vida estudados.

Considerando que no Brasil a possibilidade de triagem auditiva neonatal (TANU) e diagnóstico precoce da deficiência auditiva estão assegurados pela Lei $n^{\circ} 12.303 / 2010^{(29)}$ e Portaria $\mathrm{n}^{\circ} 587 / 2004^{\left({ }^{30}\right)}$ que institui a Política Nacional de Atenção a Saúde Auditiva (PNASA), este resultado reforça a importância dos programas de TANU estarem solidamente articulados com os programas de intervenção e a terapia fonoaudiológica que antecedem a cirurgia de implante coclear, que possibilitarão a estas crianças o uso precoce e contínuo da amplificação, culminando na melhor qualidade de vida ao longo do tempo.

A precocidade na intervenção com impacto positivo na qualidade de vida também foi demonstrada em um estudo de revisão sistemática ${ }^{(25)}$ na qual os resultados dos artigos selecionados demonstraram que crianças que realizaram cirurgia de IC em idades mais precoces apresentaram correlações estatisticamente significantes negativas com a qualidade de vida. Crianças com maior idade auditiva e com maior idade cronológica obtiveram 
correlações estatisticamente significantes positivas relativas à qualidade de vida.

Por outro lado, estudos realizaram análises comparativas entre os fatores demográficos: a idade na ativação, a idade cronológica, a idade auditiva e a qualidade de vida ${ }^{(24)}$; e a idade auditiva e a qualidade de vida de crianças com $\mathrm{IC}^{(23)}$, e não encontraram correlações estatisticamente significantes. Entretanto, no último estudo $^{(23)}$ quando realizada a comparação entre os grupos organizados de acordo com a idade auditiva (G1: crianças com idade auditiva maior que 24 meses, e G2: crianças com idade auditiva menor que 24 meses), a diferença estatisticamente significante entre os grupos confirmou a interferência positiva do tempo e do uso diário do IC sobre o domínio específico da comunicação na investigação da avaliação da qualidade de vida no grupo de crianças com maior idade auditiva.

Dos oito estudos selecionados a variável habilidades auditivas foi examinada e comparada com a qualidade de vida em sete pesquisas $^{(21-23,25-28)}$. É natural que esta variável seja amplamente estudada tendo em vista que o desenvolvimento das habilidades auditivas é apontado na literatura especializada como o principal pré-requisito para a aquisição da linguagem falada ${ }^{(3)}$, que por sua vez impacta na qualidade de vida ${ }^{(23,24,26)}$. Os estudos investigados em uma revisão sistemática da literatura ${ }^{(25)}$ revelaram que o desenvolvimento precoce das habilidades de audição apresenta relações estatisticamente significantes com as avaliações da qualidade de vida. No mesmo sentido, pesquisadores ${ }^{(26)}$ avaliaram o desenvolvimento das habilidades auditivas de crianças com IC e verificaram a presença de correlação estatisticamente significativa com o domínio comunicação na avaliação da qualidade de vida.

Outras evidências ${ }^{(27)}$ apontam a influência das habilidades auditivas sobre a qualidade de vida após o uso do IC, sem diferenças estatisticamente significativas entre os grupos de crianças usuárias de IC unilateral e usuárias de IC bilateral sequencial. No entanto, revela resultados estatisticamente significantes melhores para o grupo adaptado com IC bilateral em testes de discriminação, localização, rastreamento de movimento e percepção de fala no ruído quando comparado ao grupo com IC unilateral.

A atenção dos pesquisadores também se voltou para uma análise refinada das habilidades auditivas após a utilização de uma técnica de programação de implantes cocleares guiada por imagem, denominada Image-Guided Cochlear Implant Programming - IGCIP(22). Tal estratégia visa a análise detalhada das imagens de Tomografia Computadorizada de pacientes com IC, com o intuito de estimar a posição exata dos eletrodos na cóclea em relação ao nervo auditivo, identificar os eletrodos com altos níveis de sobreposição e estimulação, e desativá-los quando necessário. Esta técnica possibilita a criação de mapas de IC mais personalizados, promove a melhora da estimulação e, consequentemente, da percepção auditiva da fala. Os resultados do estudo $^{(22)}$ evidenciaram um aumento estatisticamente significante da qualidade de vida de crianças com IC em decorrência da melhora da percepção auditiva da fala, a partir do uso da referida técnica de programação. Ainda que se trate de uma tecnologia não utilizada em todos os centros de implante coclear, tais resultados são encorajadores para viabilizar avanços tecnológicos nas rotinas de acompanhamento de crianças implantadas, quer seja no sistema público ou privado.

Considerando que os resultados do implante coclear em crianças são multidimensionais e multifatoriais, algumas variáveis podem se manifestar fortemente na qualidade de vida de algumas crianças, e em outras não. Exemplo disto foi observado em estudos ${ }^{(21,23,28)}$, nos quais não foram encontradas correlações estatisticamente significantes entre a percepção auditiva da fala e a qualidade de vida. Ainda assim, em uma das pesquisas supracitadas ${ }^{(28)}$, quando investigada a capacidade específica de percepção da emoção vocal dos sons não linguísticos, observou-se correlação estatisticamente significante com os maiores escores de qualidade de vida.

A linguagem falada também é apontada na literatura especializada como uma das possíveis variáveis que impactam na qualidade de vida de crianças com implante coclear. $\mathrm{Na}$ presente revisão sistemática a correlação entre as habilidades de linguagem falada e a qualidade de vida de crianças com IC foi descrita por três estudos ${ }^{(25,26,28)}$. Os primeiros autores ${ }^{(26)}$ examinaram o desenvolvimento das habilidades lexicais e de fala e encontraram correlações estatisticamente significantes entre estas habilidades e os domínios da qualidade de vida. Os estudos identificados em uma revisão sistemática ${ }^{(25)}$ concordaram com tais achados e apontaram que o desenvolvimento precoce das habilidades de linguagem e comunicação se correlacionam de forma estatisticamente significante com a qualidade de vida. Por outro lado, pesquisadores ${ }^{(28)}$ que investigaram o desempenho cognitivo verbal e as habilidades de linguagem não encontraram correlações estatisticamente significantes com a qualidade de vida.

Existe uma diversidade na literatura quanto às variáveis influenciadoras e preditoras da qualidade de vida de crianças com IC. Neste estudo, a heterogeneidade das idades na cirurgia de IC, as idades auditivas e as idades cronológicas da população avaliada, bem como, a utilização de diferentes instrumentos de investigação das habilidades auditivas, de linguagem e da qualidade de vida, podem justificar a limitação no consenso entre os resultados obtidos.

Outra possível justificativa pode estar relacionada com o fato que, na população pediátrica e infantil, as avaliações da qualidade de vida ocorrem, comumente, por meio da visão dos pais, seja pela escassez de instrumentos padronizados, específicos e sensíveis à percepção das crianças, ou pela dificuldade de captação de respostas precisas neste público. Neste estudo, seis ${ }^{(21-24,26,27)}$ dos artigos identificados avaliaram a qualidade de vida das crianças a partir da perspectiva dos pais, e, apenas dois ${ }^{(25,28)}$, levaram em consideração a autopercepção das crianças. Considerando a abrangência e a subjetividade do conceito da qualidade de vida, é natural que, em alguns casos, as percepções dos pais quando comparadas às dos próprios usuários de IC, apresentem divergências. Apesar dos escassos resultados na literatura, acredita-se que, quando comparadas e/ou combinadas às percepções dos pais, o olhar das crianças sobre a sua própria qualidade de vida podem vir a contribuir com a ampliação do cenário de investigação da qualidade de vida e de suas variáveis influenciadoras.

Levando em conta a complexidade na qual envolve o processo terapêutico voltado às crianças com implante coclear, 
o fato de não haver um consenso entre os estudos selecionados não minimiza o valor dos resultados, os quais demonstraram o impacto das medidas clínicas objetivas sobre desenvolvimento subjetivo e a qualidade de vida de crianças com implante coclear, uma vez que as variáveis clínicas analisadas relativas à implantação precoce, o uso do dispositivo eletrônico, as melhores habilidades auditivas e de linguagem se correlacionaram com o aumento da qualidade de vida desta população. Compreende-se que, a complexidade do processo de indicação, implantação e acompanhamento do IC dificulta o equilíbrio esperado entre as variáveis clínicas e a qualidade de vida. Ademais, apesar dos resultados positivos descritos pela literatura, não é possível afirmar que o controle destes fatores garantirá os melhores resultados auditivos, de linguagem e de qualidade de vida. Por fim, destaca-se que, não foram encontrados estudos que investigassem a correlação entre a qualidade de vida de crianças com IC e as variáveis: permeabilidade familiar, escolaridade dos pais, e aspectos socioeconômicos, fatores estes que merecem atenção e justificam a realização de novas pesquisas na área.

\section{CONCLUSÃO}

Os fatores influenciadores que se correlacionaram com a qualidade de vida das crianças com implante coclear nos estudos selecionados foram, a implantação precoce, o uso do dispositivo eletrônico, as habilidades auditivas e as habilidades de linguagem.

Esta revisão sistemática acrescenta-se aos achados bibliográficos anteriormente publicados sobre o tema, identifica textos adicionais e sugere a relevância de mais pesquisas na área. Espera-se que este estudo auxilie na compreensão da importância do investimento sobre as variáveis influenciadoras no desenvolvimento e na qualidade de vida das crianças com IC, resultando em investimentos práticos na rotina clínica fonoaudiológica pré e pós cirúrgica destas crianças.

\section{AGRADECIMENTOS}

O presente trabalho foi realizado com apoio da Coordenação de Aperfeiçoamento de Pessoal de Nível Superior - Brasil (CAPES) - Código de Financiamento: 001, e Fundação de Amparo à Pesquisa do Estado de São Paulo (FAPESP) - Número de Processo: 2016/24216-0.

\section{REFERÊNCIAS}

1. Dunn CC, Walker EA, Oleson J, Kenworthy M, Van Voorst T, Tomblin $\mathrm{JB}$, et al. Longitudinal speech perception and language performance in pediatric cochlear implant users: the effect of age at implantation. Ear Hear. 2014;35(2):148-60. http://dx.doi.org/10.1097/AUD.0b013e3182a4a8fo. PMid:24231628.

2. Moret ALM, Costa OA. Conceituação e indicação do implante coclear. In: Boéchat EM, Menezes PL, Couto CM, Frizzo ACF, Scharlach RC, Anstasio ART, organizadores. Tratado de audiologia. São Paulo: Editora Santos; 2015. p. 327-34.

3. Colalto CA, Goffi-Gomez MVS, Magalhães ATM, Samuel PA, Hoshino $\mathrm{ACH}$, Porto BL, et al. Vocabulário expressivo em crianças usuárias de implante coclear. Rev CEFAC. 2017;19(3):308-19. http://dx.doi. org/10.1590/1982-021620171937216.
4. McKinney S. Cochlear implantation in children under 12 months of age. Curr Opin Otolaryngol Head Neck Surg. 2017;25(5):400-4. http://dx.doi org/10.1097/MOO.0000000000000400. PMid:28719394.

5. Delgado-Pinheiro EMC, Guijo LM, Bicas RCS. Interação comunicativa entre pais de crianças e adolescentes deficientes auditivos que utilizam comunicação oral. Distúrb Comun. 2014;26:743-51.

6. Fagan MK. Cochlear implantation at 12 months: limitations and benefits for vocabulary production. Cochlear Implants Int. 2015;16(1):24-31. http:/ dx.doi.org/10.1179/1754762814Y.0000000075. PMid:24954248.

7. Alegre OM, Rodríguez MC, Villar LM, Pérez D. Evaluacion de la eficacia del Implante Coclear en funcion de la edad de implantacion. Eur Sci J. 2016;12(26):42-51. http://dx.doi.org/10.19044/esj.2016.v12n26p42.

8. Phan J, Houston DM, Ruffin C, Ting J, Holt RF. Factors Affecting speech discrimination in children with cochlear implants: evidence from earlyimplanted infants. J Am Acad Audiol. 2016;27(6):480-8. http://dx.doi org/10.3766/jaaa.15088. PMid:27310406.

9. Chiossi JSC, Hyppolito MA. Effects of residual hearing on cochlear implant outcomes in children: A systematic-review. Int J Pediatr Otorhinolaryngol. 2017;100:119-27. http://dx.doi.org/10.1016/j.ijporl.2017.06.036. PMid:28802355.

10. Vieira SS, Dupas G, Chiari BM. Cochlear implant: the family's perspective. Cochlear Implants Int. 2018;19(4):216-24. http://dx.doi.org/10.1080/146 70100.2018.1426406. PMid:29363411.

11. Le Roux T, Vinck B, Butler I, Cass N, Louw L, Nauta L, et al. Predictors of pediatric cochlear implantation outcomes in South Africa. Int J Pediatr Otorhinolaryngol. 2016;84:61-70. http://dx.doi.org/10.1016/j. ijporl.2016.02.025. PMid:27063755.

12. Driver S, Jiang D. Paediatric cochlear implantation factors that affect outcomes. Eur J Paediatr Neurol. 2017;21(1):104-8. http://dx.doi. org/10.1016/j.ejpn.2016.07.012. PMid:27530431.

13. Kral A, Sharma A. Developmental neuroplasticity after cochlear implantation. Trends Neurosci. 2012;35(2):111-22. http://dx.doi.org/10.1016/j. tins.2011.09.004. PMid:22104561.

14. Ostojić S, Djoković S, Radić-šestić M, Nikolić M, Mikić B, Mirić D. Factors contributing to communication skills development in cochlear implanted children. Vojnosanit Pregl. 2015;72(8):683-8. http://dx.doi org/10.2298/VSP140221057O. PMid:26591902.

15. Zaidman-Zait A, Curle D, Jamieson JR, Chia R, Kozak FK. Health-Related Quality of Life Among Young Children With Cochlear Implants and Developmental Disabilities. Ear Hear. 2017;38(4):399-408. PMid:28207575.

16. Higgns JPT, Green S. Cochrane handbook for systematic reviews of interventions. Version 5.1.0. The Cochrane Collaboration. 2011.

17. Moher D, Liberati A, Tetzlaff J, Altman DG, PRISMA Group. Preferred reporting items for systematic reviews and meta-analyses: the PRISMA Statement. Int J Surg. 2010;8(5):336-41. http://dx.doi.org/10.1016/j. ijsu.2010.02.007. PMid:20171303.

18. Urrútia G, Bonfill X. Declaración PRISMA: una propuesta para mejorar la publicación de revisiones sistemáticas y metanálisis. Med Clin (Barc). 2010;135(11):507-11. http://dx.doi.org/10.1016/j.medcli.2010.01.015. PMid:20206945.

19. ASHA: American Speech-Language-Hearing Association. An introduction to clinical trials [Internet]. Rockville: American Speech-Language-Hearing Association; 2005 [citado em 2018 Mai 21]. Disponível em: http://www. asha.org/Publications/leader/2005/050524/f050524a/

20. OMS: Organização Mundial da Saúde. The World Health Organization Quality of Life Assessment (WHOQOL): Position paper from the World Health Organization. Soc Sci Med. 1995;41(10):1404. PMid:8560308.

21. Liu H, Liu HX, Kang HY, Gu Z, Hong SL. Evaluation on health-related quality of life in deaf children with cochlear implant in China. Int J Pediatr Otorhinolaryngol. 2016;88:136-41. http://dx.doi.org/10.1016/j. ijporl.2016.06.027. PMid:27497401.

22. Noble JH, Hedley-Williams AJ, Sunderhaus L, Dawant BM, Labadie RF, Camarata SM, et al. Initial results with image-guided cochlear implant programmining in children. Otol Neurotol. 2016;37(2):63-9. http://dx.doi. org/10.1097/MAO.0000000000000909. PMid:26756157. 
23. Almeida RP, Matas CG, Couto MIV, Carvalho ACM. Avaliação da qualidade de vida em crianças usuárias de implante coclear. CoDAS. 2015;27(1):2936. http://dx.doi.org/10.1590/2317-1782/20152014129. PMid:25885194.

24. Kumar R, Warner-Czyz A, Silver CH, Loy B, Tobey E. American parent perspectives on quality of life in pediatric cochlear implant recipients. Ear Hear. 2015;36(2):269-78. http://dx.doi.org/10.1097/AUD.0000000000000108. PMid:25377531.

25. Morettin M, Santos MJD, Stefanini MR, Antonio FL, Bevilacqua MC, Cardoso MRA. Measures of quality of life in children with cochlear implant: systematic review. Braz J Otorhinolaryngol. 2013;79(3):382-90. http://dx.doi.org/10.5935/1808-8694.20130066. PMid:23743756.

26. Fortunato-Tavares T, Befi-Lopes DM, Bento RF, Andrade CRF. Crianças com implante coclear: habilidades comunicativas e qualidade de vida. Rev Bras Otorrinolaringol (Engl Ed). 2012;78:15-25. http://dx.doi.org/10.1590/ S1808-86942012000100003.

27. Lovett RE, Kitterick PT, Hewitt CE, Summerfield AQ. Bilateral or unilateral cochlear implantation for deaf children: an observational study. Arch Dis Child. 2010;95(2):107-12. http://dx.doi.org/10.1136/adc.2009.160325. PMid:19948510.

28. Schorr EA, Roth FP, Fox NA. Quality of life for children with cochlear implants: perceived benefits and problems and the perception of single words and emotional sounds. J Speech Lang Hear Res. 2009;52(1):141-52. http://dx.doi.org/10.1044/1092-4388(2008/07-0213). PMid:18664684.

29. Brasil. Lei $n^{\circ} 12.303$, de 2 de agosto de 2010. Dispõe sobre a obrigatoriedade de realização do exame denominado Emissões Otoacústicas Evocadas. Diário Oficial da União; Brasília; 2 ago. 2010.

30. Brasil. Portaria $\mathrm{n}^{\circ}$ 587, de 07 de outubro de 2004. Dispõe sobre a organização e a implantação das Redes Estaduais de Atenção à Saúde Auditiva. Diário Oficial da União; Brasília; 7 out. 2004.

\section{Contribuição dos autores}

JMS é a autora principal do trabalho e realizou a concepção do projeto do estudo, aquisição, análise e interpretação dos dados, elaboração do artigo, correção, revisão crítica para conteúdo intelectual relevante e aprovação final da versão a ser apresentada para publicação. ALMM foi a orientadora responsável pela orientação nas etapas de elaboração do projeto, aquisição, análise e interpretação dos dados, correção do artigo revisão crítica para conteúdo intelectual relevante e aprovação final da versão a ser apresentada para publicação. PDC auxiliou na concepção do projeto do estudo, elaboração do artigo, correção, revisão crítica para conteúdo intelectual relevante e aprovação final da versão a ser apresentada para publicação. 